\title{
Molecular architecture of the flagellar export apparatus reveals membrane remodeling and conformational changes crucial for flagellar assembly
}

\author{
Brittany Carroll ${ }^{1}$, Md Motaleb $^{2}$ and Jun Liu ${ }^{1}$ \\ ${ }^{1}$ Yale University, United States, ${ }^{2}$ East Carolina University, United States
}

\section{Background}

A highly conserved export apparatus, powered by the proton motive force (PMF) and ATP hydrolysis, is required to assemble bacterial flagella. The flagellar export apparatus consists of five integral membrane proteins (FlhA, FlhB, FliP, FliQ, and FliR) and three cytoplasmic proteins (FliH, FliI, and FliJ)(Macnab, 2004). The membrane components form an export gate for secretion of substrates, while the three cytoplasmic proteins form an ATPase complex that promotes the export process by binding and delivering substrates to the export gate. In order to determine the molecular events that govern the export of substrates, it is essential to understand the subunit organization of the intact export apparatus. FlhA is the largest membrane protein essential for coupling proton flow with the translocation of substrates into the central channel of the growing flagellum (Kihara et al., 2001; Minamino et al., 2010; Zhu et al., 2002). FlhA consists of an N-terminal transmembrane domain (FlhATM) and a C-terminal cytoplasmic domain (FlhAC)(McMurry et al., 2004). A relatively small conserved cytoplasmic domain of FlhA (termed the FHIPEP domain) is also critical in Salmonella (Erhardt et al., 2017). FlhB, required for substrate specificity, regulates hook length and switching to flagellin secretion for filament assembly via an autocleavage event (Ferris et al., 2005; Fraser et al., 2003). FliP, FliQ, and FliR form the core complex (FliPQR) involved in protein secretion (Kuhlen et al., 2018). Cryo-EM structures of the purified FliPQR and FlhB complexes reveal an unexpected topology and orientation of the complex, with no canonical transmembrane regions. Instead, they form a helical structure that sits at the base of the basal body, mainly in the periplasm (Kuhlen et al., 2018; Kuhlen et al., 2020). Furthermore, cryo-electron tomography (cryo-ET) studies revealed that the cytosolic ATPase complex forms a hub-and--spoke structure attached directly to the flagellar C-ring (Qin et al., 2018)(Lin et al., 2015). Despite extensive structural characterization of the key protein components by X-ray crystallography, cryo-EM and cryoET, the subunit organization of the intact export apparatus remains incomplete.

\section{Methods}

We deployed cryo-ET and sub-tomogram averaging to visualize the intact export apparatus of periplasmic flagella in Borrelia burgdorferi, the causative agent of Lyme disease. In particular, in situ structure of the intact export apparatus is compared with those derived from specific export apparatus mutants.

\section{Results}

We determined high resolution in situ structure of the intact flagellar export apparatus in B. burgdorferi. Using export gate protein deletion mutants, we reveal that the inner membrane undergoes major remodeling to accommodate the assembly of the FliPQR complex (Figure). We provided evidence that the membrane remodeling is required for the assembly of the FlhA ring. Using two specific point mutants in FlhA, we showed how disruption of proton transport causes improper flagellar assembly.

\section{Conclusions}

We report that the FliPQR complex facilitates membrane remodeling, which is essential for the assembly of the FlhA ring and the formation of the export gate. We resolved full-length FlhA, resulting in identification of two nonameric rings connected by tubular density. Furthermore, we provided direct evidence that flagellar substrate exportation is dependent on the proton channel of FlhA and conformational change of the FlhA ring.

\section{Acknowledgments}

We thank Jennifer Aronson for proofreading. This work was supported by grants R01AI087946 and R01AI132818 from National Institutes of Health. 

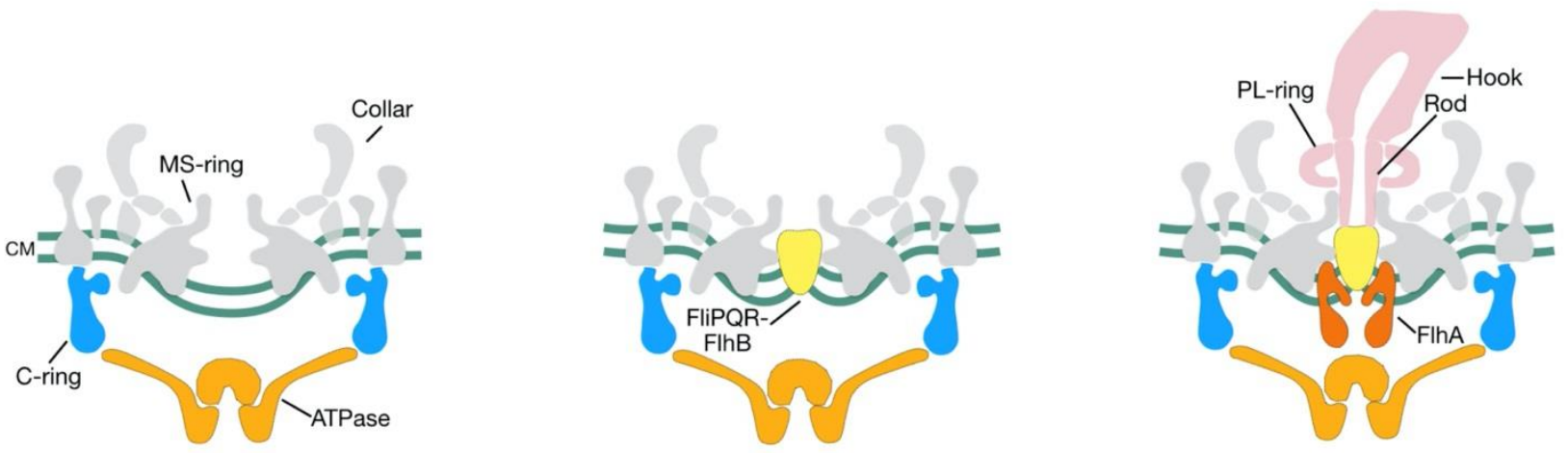

Figure 1. Figure. Models of the membrane-bound B. burgdorferi flagellar motor in three distinct conformations: single concave membrane (left) in the absence of the export gate complex, pinched membrane (middle) with the assembly of the FliPQR-FlhB complex, and FlhA docking in the pinched membrane (right). The membrane remodeling is critical for FlhA assembly and function.

\section{References}

Erhardt, M., et al. (2017). Mechanism of type-III protein secretion: Regulation of FlhA conformation by a functionally critical charged-residue cluster. Mol Microbiol 104, 234-249.

Ferris, H.U., et al. (2005). FlhB regulates ordered export of flagellar components via autocleavage mechanism. J Biol Chem 280, 41236-41242.

Fraser, G.M., et al. (2003). Substrate specificity of type III flagellar protein export in Salmonella is controlled by subdomain interactions in FlhB. Mol Microbiol 48, 1043-1057.

Kihara, M., et al. (2001). Intergenic suppression between the flagellar MS ring protein FliF of Salmonella and FlhA, a membrane component of its export apparatus. J Bacteriol 183, 1655-1662.

Kuhlen, L., et al. (2018). Structure of the core of the type III secretion system export apparatus. Nat Struct Mol Biol 25, 583-590.

Kuhlen, L., et al. (2020). The substrate specificity switch FlhB assembles onto the export gate to regulate type three secretion. Nat Commun 11, 1296.

Lin, T., et al. (2015). Mutations in the Borrelia burgdorferi Flagellar Type III Secretion System Genes fliH and fliI Profoundly Affect Spirochete Flagellar Assembly, Morphology, Motility, Structure, and Cell Division. MBio 6.

Macnab, R.M. (2004). Type III flagellar protein export and flagellar assembly. Biochimica et biophysica acta 1694, 207-217.

McMurry, J.L., et al. (2004). Analysis of the cytoplasmic domains of SalmonellaFlhA and interactions with components of the flagellar export machinery. J Bacteriol 186, 7586-7592.

Minamino, T., et al. (2010). Role of the C-terminal cytoplasmic domain of FlhA in bacterial flagellar type III protein export. J Bacteriol 192, 1929-1936.

Qin, Z., et al. (2018). Cryo-electron tomography of periplasmic flagella in Borrelia burgdorferi reveals a distinct cytoplasmic ATPase complex. PLoS Biol 16, e3000050.

Zhu, K., et al. (2002). Interactions among membrane and soluble components of the flagellar export apparatus of Salmonella. Biochemistry 41, 9516-9524. 